Fisioter Bras 2020;21(4):334-5

https://doi.org/10.33233/fb.v21i4.4318

\title{
EDITORIAL \\ Reabilitação de pacientes sobreviventes ao COVID-19: O próximo desafio
}

Marco Orsini ${ }^{1}, 2$, Jacqueline Stephanie Fernandes do Nascimento ${ }^{1}$, Nicolle dos Santos Moraes Nunes ${ }^{1}$, Janie Kelly Fernandes do Nascimento ${ }^{1}$, Renata Rodrigues Teixeira de Castro ${ }^{1}$, Marco Antônio Alves Azizi ${ }^{1}$, Mauricio de Sant' Anna $\mathrm{Jr}^{3}$

1 Universidade Iguaçu, Escola de Medicina, RJ, Brasil

2Universidade de Vasssouras, RJ, Brasil

${ }^{3}$ Instituto Federal, Ciência e Tecnologia do Rio de Janeiro, RJ, Brasil (IFRJ)

Correspondência: Mauricio de Sant Anna Jr, Instituto Federal de Educação, Ciência e Tecnologia do Rio de Janeiro, Campus Realengo, Rua Professor Carlos Wenceslau, 343 Realengo 21715-000

Marco Orsini: orsinimarco@hotmail.com Jacqueline Stephanie Fernandes do Nascimento: jac.fn@hotmail.com Nicolle dos Santos Moraes Nunes: nicolle.nunes_@hotmail.com.br Janie Kelly Fernandes do Nascimento: janiekelly@hotmail.com

Renata Rodrigues Teixeira de Castro: castrorrt@gmail.com

Marco Antônio Alves Azizi: marcoazizi@gmail.com

Mauricio de Sant' Anna Jr: mauricio.junior@ifrj.edu.br

Em dezembro de 2019, um surto de pneumonia de etiologia desconhecida foi relatado em Wuhan, província de Hubei, China. A maioria desses casos estava epidemiologicamente relacionada ao mercado atacadista de frutos do mar de Huanan [1]. A inoculação do líquido obtido por lavagem broncoalveolar de pacientes com essa pneumonia levou ao isolamento de um novo coronavírus, inicialmente chamado 2019-nCov, e posteriormente denominado síndrome respiratória aguda grave pelo coronavírus 2 (SARS-CoV-2) [2].

Devido ao aumento do número de casos de COVID-19 em todo o mundo, foi decretado pela Organização Mundial da Saúde, um estado de pandemia. O COVID-19 é uma doença que pode causar uma importante lesão pulmonar, exigindo hospitalização para uso de oxigênio e, no último caso, internação em unidade de terapia intensiva com uso de ventilação mecânica invasiva $[2,3]$.

Pacientes que sobrevivem ao evento agudo causado pelo COVID-19 costumam sofrer os efeitos deletérios de uma internação prolongada, como: alterações cognitivas, depressão, ansiedade, alterações de mobilidade, além de alterações cardiovasculares e pulmonares.

Como essa condição é real em todo o mundo, qual será nosso próximo desafio? Este é um segundo momento da pandemia e que merece ser tratado com a mesma atenção pelas autoridades e que levou a Organização Pan-Americana da Saúde a publicar um documento intitulado Rehabilitation considerations during the COVID-19 outbreak [4].

Este documento deixa claro o papel da reabilitação no acompanhamento a longo prazo de pacientes com COVID-19.

Muitos foram os relatos descrevendo o processo de reabilitação em pacientes sobreviventes ao COVID-19, em atendimento ambulatorial. Yang e Yang [5] publicaram um artigo de revisão mencionando os benefícios da reabilitação pulmonar para pacientes afetados pelo COVID-19. Sheehy [6] também expressou sua opinião sobre a importância da reabilitação em sobreviventes do COVID-19, no entanto, vale ressaltar que esses dois autores (assim como outros que não foram mencionados aqui) ainda estavam no campo de hipóteses e elucubrando alternativas.

De todos os artigos publicados presente até o momento sobre essa temática, um que merece destaque é elegante trabalho realizado por Liu et al. [7] pelo fato de ser pioneiro em alguns apontamentos. Os autores descreveram os efeitos de um programa de reabilitação com duração de seis semanas em pacientes sobreviventes ao COVID-19 e observaram melhoras 
significativas na função pulmonar, por meio da espirometria, melhora da capacidade funcional mensurada através do teste de caminhada de seis minutos, além de melhoria na qualidade de vida através do questionário SF-36, atividades da vida diária através da Medida de Independência Funcional (MIF).

Cabe ressaltar que, em relação à reabilitação de pacientes sobreviventes do COVID-19, as informações começam a ser organizadas para facilitar o desempenho dos profissionais de saúde. Com base nessa premissa, o de Stanford Hall para a reabilitação pós-COVID-19 [8] realizado por pesquisadores do Reino Unido reúne até agora as melhores evidências para a condução da reabilitação nesse novo cenário que se apresenta a todo o mundo. O documento abrange recomendações para: reabilitação pulmonar, cardiovascular, neuromusculoesquelética e neurológica, sem deixar de lado as recomendações médicas e psicológicas, diretamente relacionadas à reabilitação.

A reabilitação sempre foi descrita como um processo multidisciplinar e, em pacientes sobreviventes ao COVID-19, devido ao fato de ser uma condição clínica e funcional de recente descoberta, será extremamente importante para os profissionais trocarem o máximo de informações possíveis, para que os indivíduos retomem as suas atividades diárias, o mais próximo possível do que eram anteriormente.

O documento Rehabilitation considerations during the COVID-19 outbreak [4] é enfático ao afirmar que os esforços do governo para oferecer reabilitação aos pacientes que sobrevivem ao COVID-19 devem ser os mesmos realizados quanto a oferta de leitos hospitalares.

Além dos centros de reabilitação, dos programas ambulatoriais, dos atendimentos domiciliares, a pandemia nos mostrou uma potencialidade pertinente ao momento atual, a telessaúde, incluindo telemedicina e tele-reabilitação.

\section{Referências}

1. Ciotti M, Angeletti S, Minieri M, Giovannetti M, Benvenuto D, Pascarella S COVID-19 outbreak: an overview. Chemotherapy 2020;64(5/6):215-23.

https://doi.org/10.1159/000507423

2. Zhu N, Zhang D, Wang W, Li X, Yang B, Song J et al. China novel coronavirus investigating and research team. A novel coronavirus from patients with pneumonia in china, 2019. N Engl J Med 2020;382(8):727-33.

https://doi.org/10.1056/NEJMoa2001017

3. Huang $X$, Wei F, Hu L, Wen L, Chen K. Epidemiology and clinical characteristics of COVID-19. Arch Iran Med 2020;1;23(4):268-71. https://doi.org/10.34172/aim.2020.09

4. Pan American Health Organization: Rehabilitation considerations during the COVID-19 outbreak. 28 de abril de 2020. Disponível em:

https://www.paho.org/en/documents/rehabilitation-considerations-during-covid-19outbreak

5. Yang L, Yang T. Pulmonary rehabilitation for patients with coronavirus disease 2019 (COVID-19). Chronic Dis Transl Med 2020. https://doi.org/10.1016/i.cdtm.2020.05.002

6. Sheehy LM. Considerations for postacute rehabilitation for survivors of COVID-19. JMIR Public Health Surveill 2020 8;6(2):e19462. https://doi.org/10.2196/19462

7. Liu K, Zhang W, Yang Y, Zhang J, Li Y, Chen Y. Respiratory rehabilitation in elderly patients with COVID-19: A randomized controlled study. Complement Ther Clin Pract 2020;39:101166. https://doi.og/10.1016/i.ctcp.2020.101166

8. Barker-Davies RM, O'Sullivan O, Senaratne KPP, Baker P, Cranley M, Dharm-Datta S. The Stanford Hall Consensus Statement for post-COVID-19 Rehabilitation. Br J Sports Med 2020;54:959-69. https://doi.org/10.1136/bjsports-2020-102596 